\title{
Enhancing the Role of Electric Vehicles in the Power Grid: Field Validation of Multiple Ancillary Services
}

Knezovic, Katarina; Martinenas, Sergejus; Andersen, Peter Bach; Zecchino, Antonio; Marinelli, Mattia

Published in:

IEEE Transactions on Transportation Electrification

Link to article, DOI:

10.1109/TTE.2016.2616864

Publication date:

2016

Document Version

Peer reviewed version

Link back to DTU Orbit

Citation (APA):

Knezovic, K., Martinenas, S., Andersen, P. B., Zecchino, A., \& Marinelli, M. (2016). Enhancing the Role of Electric Vehicles in the Power Grid: Field Validation of Multiple Ancillary Services. IEEE Transactions on Transportation Electrification, 3(1), 201 - 209. https://doi.org/10.1109/TTE.2016.2616864

\section{General rights}

Copyright and moral rights for the publications made accessible in the public portal are retained by the authors and/or other copyright owners and it is a condition of accessing publications that users recognise and abide by the legal requirements associated with these rights.

- Users may download and print one copy of any publication from the public portal for the purpose of private study or research.

- You may not further distribute the material or use it for any profit-making activity or commercial gain

- You may freely distribute the URL identifying the publication in the public portal 


\title{
Enhancing the Role of Electric Vehicles in the Power Grid: Field Validation of Multiple Ancillary Services
}

\author{
Katarina Knezović, Student Member, IEEE, Sergejus Martinenas, Student Member, IEEE, Peter Bach \\ Andersen, Member, IEEE, Antonio Zecchino, Student Member, IEEE, Mattia Marinelli, Member, IEEE
}

\begin{abstract}
With increased penetration of distributed energy resources and electric vehicles (EVs), different EV integration strategies can be used for mitigating various adverse effects, and supporting the grid. However, the research regarding EV smart charging has mostly remained on simulations, whereas the experimental validation has rarely been touched upon. This paper focuses mainly on evaluating the technical feasibility of a series-produced EV to provide flexibility in real distribution grids. The implemented controller uses contemporary and widely supported standards for limiting the EV charging rate, which essentially means it is applicable to any EV complying with IEC 61851 and SAE J1772 standards. The field test validation is conducted in a real Danish distribution grid with a Nissan Leaf providing three ancillary services through unidirectional AC charging, namely congestion management, local voltage support, and primary frequency regulation. Several performance parameters, such as EV response time and accuracy, are assessed and benchmarked with current requirements. Ultimately, the paper aims to strengthen the applied research within the EV integration domain through validating smart grid concepts on original standard-compliant equipment.
\end{abstract}

Index Terms-ancillary services, electric vehicle, power distribution control, power system testing, smart charging

\section{INTRODUCTION}

$\mathbf{F}$ UNDAMENTAL changes occurring in the electric power system promoted by the global sustainability efforts have started to reshape the grid operation. With increased penetration of distributed energy resources, such as photovoltaic installations (PVs) and various electric vehicles (EVs) [1], [2], there is an increased need for control strategies which would allow them to provide flexibility services. EVs seem to be one of the eminent resources for providing various services due to their defining properties: (1) they are a large load compared to other residential loads, (2) they have quick-response with potential bi-directional power flow capabilities, and (3) they are available most of the time with high degree of flexibility [3], [4]. Significant amount of research has been done to address the arising EV challenges as well as to capture their benefits [5], [6].

Overall, the literature pointed out that EVs can have high potential in providing regulation services to the transmission system operator (TSO), especially primary frequency regulation, due to their rapid response. Ref. [7]

The authors are with the Centre for Electric Power and Energy, Department of Electrical Engineering, Technical University of Denmark (DTU) Roskilde, Denmark (e-mail: kknez@elektro.dtu.dk; smar@elektro.dtu.dk; pba@elektro.dtu.dk; antozec@elektro.dtu.dk; matm@elektro.dtu.dk). concluded that EV participation in regulation markets offers a substantial earning potential to the EV owners, whereas [8]-[10] showed that EVs with different droop controls can be effective in primary frequency control, likewise in larger systems and isolated microgrids. On the other hand, considering that residential EV charging highly impacts the local grid, different strategies are proposed for EVs providing flexibility to the distribution system operator (DSO), namely congestion management and voltage regulation [11], [12]. It is shown across a variety of studies that centralized EV control reduces losses, improves voltage stability and performs peak shaving or congestion control [13]-[15]. On the other hand, decentralized control based only on local measurements provides similar results [16]. Additionally, more and more countries, among which Germany and Italy, request small inverter-interfaced PVs to provide reactive power. Similarly, since EVs are also inverter-based, their electronic equipment could potentially enable reactive power exchange with the grid without affecting the active power flow, provided the inverter is properly sized [17], [18].

Even though the identified literature analysed different EV control strategies, it mostly remained on simulations, whereas the experimental validation is rarely touched upon. Ref. [19] tested the developed smart charging algorithm on a commercial EV, but focused only on minimising the charging cost, not on providing any ancillary services. In general, when dealing with ancillary services, the literature assumes an ideal EV response to the control signal, and omits response latencies and inaccuracies which may greatly impact the results. The importance of hardware-in-the-loop for evaluating the ancillary service provision of inverter-interfaced DERs is discussed in [20]. The works described in [21] experimentally tested proposed frequency control, but the $\mathrm{EV}$ was represented by a custom-made set of Li-Ion batteries whose behaviour differs from commercial EVs. On the other hand, [22] uses series-produced EVs for experimental validation, but only for frequency control, and in laboratory environment. Thus, an extensive experimental activity is required to prove the feasibility of different EV controls with contemporary technology and series-produced cars outside the laboratory environment.

The main contributions of this manuscript can be summarized as follows:

- Validating the developed EV smart charging controller for providing multiple ancillary services, i.e., 
congestion management, local voltage support, and frequency-controlled normal operation reserve (primary frequency regulation).

- Assessing the technical feasibility of such a controller with currently available technology and series produced vehicle. Implemented controller uses contemporary standards for limiting the EV charging rate, which essentially means it can be used with all EVs compliant with IEC 61851 [23] and SAE J1772 [24] amounting to 7563 only in Denmark at the end of 2015 [25]. Assuming $50 \%$ service participation rate with \pm 5 A flexibility per vehicle, this results in approximately $\pm 4 \mathrm{MW}$ of available system flexibility.

- Conducting a field validation in a real distribution grid with no controllability over other residential units, and limited amount of measurement equipment.

- Investigating issues which may arise when dealing with the practical implementation of EVs providing ancillary services, i.e. several performance parameters such as vehicle responsiveness and accuracy to compare the fulfilment with the existing requirements.

The paper is structured as follows. Section I presented the contemporary standards and literature survey regarding the tested services. Further on, Section II presents the implemented control strategy. The description of the experimental field setup, the performed test activities, and the parameters for result evaluation are given in Section III. Finally, the results are presented and discussed in Section IV followed by the conclusions in Section V.

\section{EV SMART CHARGING CONTROLLER}

\section{A. Control logic for various ancillary services}

To validate the technical feasibility of contemporary EVs providing various ancillary services, a universal smart charging controller was developed, which is applicable to any EV compliant with IEC 61851 and SAE J1772. This controller can be used for performing centralised EV control such as congestion management and primary frequency control, or as an autonomous controller implemented directly in the Electric Vehicle Supply Equipment (EVSE) for local voltage regulation.

The control logic itself is based on droop control whose characteristics have been inspired by the corresponding service requirements, and the current EV capabilities. The droop control is a well-established control scheme commonly used in the power system domain due to its simplicity, which makes it a viable solution for EV flexibility provision. As shown in [9], [10], [26], [27], EVs equipped with a droop control can provide primary frequency regulation, and maintain the system frequency, both in the case of centralised and decentralised strategies likewise in an islanded mode or when grid connected. Moreover, it has been shown that droop control can be efficiently applied to EVs providing voltage regulation and congestion management [16], [28], [29] in order to support the local distribution grid. When utilising decentralised droop control for local support, the control performance is guaranteed as long as the physical properties

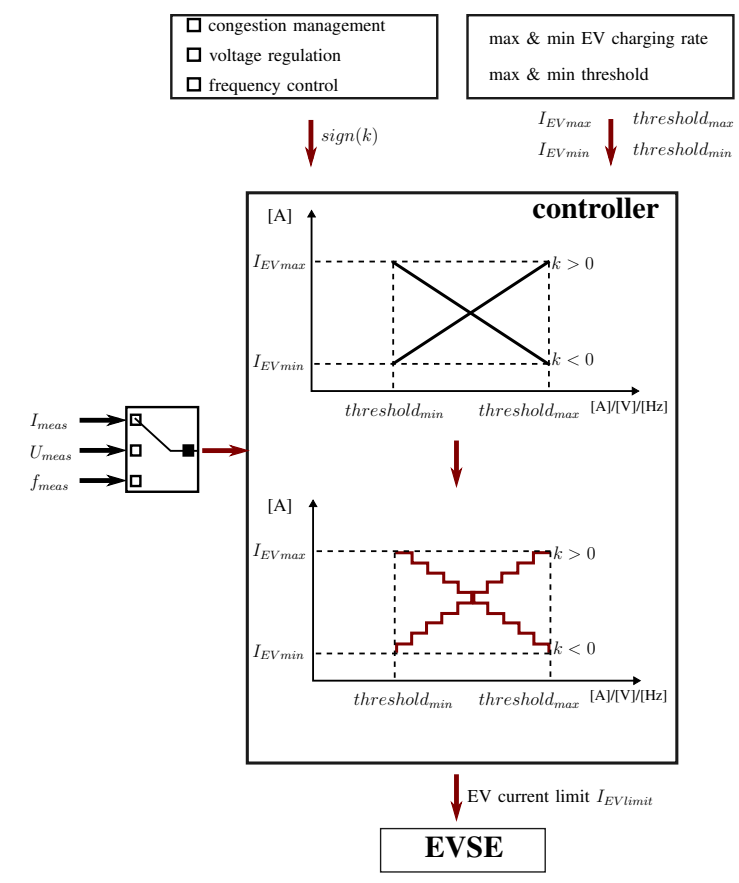

Fig. 1: Schematic overview of controller's input parameters for droop characteristics construction.

of the system do not change which is usually the case with radial distribution grids.

In the developed controller, one can easily switch between the services by choosing the measurement to which the EV is responding, and changing the droop characteristics as desired. As shown in Fig. 1, the specified input parameters construct the ideal, and the effective EV droop characteristics which is dependent on the current EV capabilities. The necessary input parameters are: (1) the type of service which defines the droop characteristic sign $(\operatorname{sign}(k))$ and the input measurement (MV-LV transformer loading $I_{\text {meas }}$, local phase-to-neutral voltage $U_{\text {meas }}$ or system frequency $f_{\text {meas }}$ ), (2) the minimum $\left(I_{E V \min }\right)$ and the maximum EV charging current $\left(I_{E V \max }\right)$, and (3) the minimum (threshold ${ }_{m i n}$ ) and the maximum threshold (threshold tmax $_{\text {) }}$ ) for the chosen service.

First of all, according to contemporary standards [23], [24], all EVs must be able to limit their charging rate between the minimum charging current of $6 \mathrm{~A}$ and the maximum one, which is the EVSE rated current. These values are the ones used in the controller if not specified otherwise. The same standards also require that the charging rate is limited in discrete 1 A steps, whereas the response to intermediate currents is not guaranteed. Hence, the effective EV droop characteristic cannot be linear like the ideal theoretical one due to the described practical limitations. The defined minimum $\left(I_{E V \min }\right)$ and the maximum EV charging currents $\left(I_{E V \max }\right)$ specify the band within which the EV charging rate $I_{E V l i m i t}$ can be controlled for all services as follows:

$$
\begin{gathered}
I_{E V \min } \leq I_{\text {EVlimit }} \leq I_{E V \max } \\
I_{\text {EVlimit }} \in \mathbb{N}
\end{gathered}
$$

For a typical 16 A single-phase EVSE, 11 current steps are available in total (i.e., $6 \mathrm{~A}, 7 \mathrm{~A}, \ldots, 16 \mathrm{~A}$ ). In case the grid 
components are not sized for the maximum charging rate, e.g. if residential fuses are $13 \mathrm{~A}$, the maximum rate can be limited within the controller. Naturally, this results in a lower EV current span available for flexibility provision. Moreover, in case an aggregator would like to realize an ideal linear behaviour for a specific service, it would be necessary to have a sufficient amount of EVs so that, once aggregated, they show an equivalent linear response. Nevertheless, the number of current steps does not influence the EV performance evaluation in terms of response time and accuracy.

Secondly, depending on the specified service, the controller responds to the different measurement data as explained in II-B. Regardless of the chosen service, the range threshold ${ }_{\text {min }}-$ threshold $_{\max }$, within which the EV provides flexibility, has to be defined. This range is either the transformer loading, the voltage, or the frequency range. The set thresholds are arbitrarily chosen, and can be either constant or varying depending on the time of the day and the specific grid circumstances. It is up to the system operator to determine the most suitable thresholds depending on the distribution grid characteristics. Since the thresholds are defined within the control logic, they can also be dynamically changed if an adaptive droop characteristic is required, or if the droop characteristic is to be periodically updated to include the EV SOC target. The process of threshold choice can be automatised with estimation techniques, but designing an adaptive control logic was not the main focus point of this paper, so the thresholds are set to fixed values. Similarly as derived in [28], here the EV charging rate is a linear characteristic of the input measurement data, and can generally be calculated as the multiplication between the droop gain, and the difference between the measured and the nominal value (i.e., current, voltage or frequency). Hence, once the thresholds are defined, the droop slope $k$ is calculated as follows:

$$
k=\frac{I_{E V \min }-I_{E V \max }}{\text { threshold }_{\min }-\text { threshold }_{\max }}
$$

One should note that the droop characteristic will be positive in case of voltage and frequency regulation, i.e., the EV charging rate linearly increases if the voltage or the frequency increases, whereas it is the opposite for congestion management where the EV charging rate linearly decreases if the transformer current increases. Therefore, the EV charging current limit $I_{\text {calc }}$ is calculated according to equation (3) for voltage regulation, equation (4) for frequency control, and equation (5) for the congestion management. Since the set EV charging limit must be an integer value due to the practical limitations set by the standards, the calculated current is rounded up.

$$
\begin{aligned}
I_{\text {calc }} & =\left\lceil+k \cdot\left(U_{\text {meas }}-\text { threshold }_{\max }\right)+I_{E V \max }\right\rceil \\
I_{\text {calc }} & =\left\lceil+k \cdot\left(f_{\text {meas }}-\text { threshold }_{\text {max }}\right)+I_{E V \max }\right\rceil \\
I_{\text {calc }} & =\left\lceil-k \cdot\left(I_{\text {meas }}-\text { threshold }_{\text {min }}\right)+I_{E V \max }\right\rceil
\end{aligned}
$$

Then, the EV charging limit $I_{\text {EVlimit }}$ is set as:

$$
I_{\text {EVlimit }}= \begin{cases}I_{\text {calc }}, & I_{E V \min } \leq I_{\text {calc }} \leq I_{E V \max } \\ I_{E V \max }, & I_{\text {calc }}>I_{E V \max } \\ I_{E V \min }, & I_{\text {calc }}<_{E V \min }\end{cases}
$$

The specific input parameters chosen for the experimental validation will be explained in Section III.

The droop control logic is chosen due to its simplicity which makes it cheap and applicable on wide range of computing devices. However, the developed controller can be extended for other control strategies as well, e.g., multi-agent systems [30], [31], where the EV charging limit is calculated based on different input signals such as the market price, as well as for a more complex droop control strategies which include the user preferences [32]. Naturally, for a more complex control logic, the overall performance could decrease due to a longer computational time. Experimental investigation of such strategies has been left for future work.

\section{B. Communication architecture}

The communication architecture for the implemented smart charging controller is shown in Fig. 2.

Depending on the chosen service, input for the control logic comes from a different measurement device. The Smart Grid Unit (SGU) installed at the transformer substation sends the single-phase current measurements $I_{\text {meas }}$ via the Internet, similarly to the DEIF MTR-3 device which measures the system frequency $f_{\text {meas }}$. These devices could be replaced with any measurement device capable of sending the data via the Internet. On the other hand, the local phase-to-neutral voltage measurement $U_{\text {meas }}$ comes from the DEIF MIC-2 device installed in the EVSE, which is connected to the control logic by Ethernet using the MODBUS protocol. The actual measurements are polled using the corresponding data poller subroutines within the controller. The control logic actuates the EV charging power by setting the appropriate current limit in the EVSE controller located within the EVSE, whereas the EV itself is connected to the EVSE using the IEC 61851 standard. According to this standard, the EV listens to the EVSE communication line (called the Control Pilot line), in the form of a Pulse Width Modulation (PWM) signal whose duty cycle indicates the maximum EV charging limit.

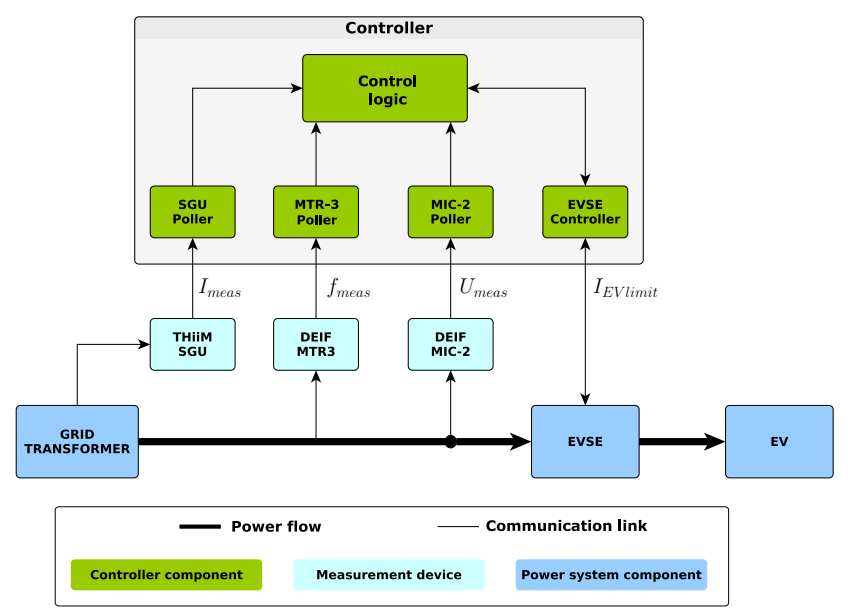

Fig. 2: Communication architecture diagram for the tested smart charging controller. 
The described architecture can easily be extended to more EVs, both for centralised and decentralised algorithms. In case of a centralised strategy, the control logic would just be connected to several others EVSEs which would allow the control of large EV amounts. On the other hand, the shown architecture would be implemented within each individual EVSE in case of decentralised control, or in systems based on autonomous agents. Since the developed controller is based only on contemporary standards and equipment, it can easily be integrated in the current power system under the smart grid concept.

\section{EXPERIMENTAL FIELD TEST}

The field test was conducted in a $400 \mathrm{~V}$ distribution feeder located in the suburban area of southern Zealand, Denmark, whose topology is depicted in Fig. 3. This feeder is radially run and connected to the MV network through a typical $400 \mathrm{kVA}$ transformer. It consists of 43 residential houses with a three-phase grid connection, and a common neutral conductor grounded at the transformer substation. There are three additional feeders under the same transformer station with approximately the same number of houses per feeder. For the conducted field trials, the EV was connected to a standard Schuko plug in a residential house located towards the end of the feeder at phase $c$ of node 612. As depicted in Fig. 3, the field test setup consists of the following components:

- series produced EV (Nissan Leaf) with $24 \mathrm{kWh}$ Li-Ion battery and single-phase $16 \mathrm{~A}(230 \mathrm{~V})$ connection,

- EVSE with PhoenixContact controller for limiting the EV charging current,

- ThiiM Smart Grid Unit (SGU) for transformer current measurements (located at the transformer substation) with 0.1 A accuracy and 30 second sampling rate,

- DEIF MIC-2 for local phase-to-neutral voltage measurements and EV current measurement with $0.5 \%$ accuracy and 1 second sampling rate,

- DEIF MTR-3 for frequency measurements (located at Ris $\varnothing$ Campus, Technical University of Denmark) with 10 $\mathrm{mHz}$ accuracy and 1 second sampling rate, and

- notebook with Internet connection for receiving the measurements and running the control logic.

Moreover, one should note that none of the other residential loads were controlled, so the consumption variability comes solely from the users themselves.

The three used droop characteristics are shown in Fig. 4, respectively for congestion management, voltage regulation, and frequency-controlled normal (FCN) operation reserve. Since the field experiment was conducted in a real residential house whose fuses are not sized for such a heavy load, the maximum EV charging rate was set to $12 \mathrm{~A}$ which resulted in seven possible charging current for all services, seen as six steps in Fig. 4. The thresholds for each service have been chosen as follows: (1) $I_{\min }=90 \mathrm{~A}$ and $I_{\max }=120 \mathrm{~A}$ for congestion management, (2) $U_{\min }=0.96 U_{n}$ and $U_{\max }=$ $0.98 U_{n}$ for voltage support, and (3) $f_{\min }=49.9 \mathrm{~Hz}$ and $f_{\text {max }}=50.1 \mathrm{~Hz}$ for frequency-controlled normal operation reserve.

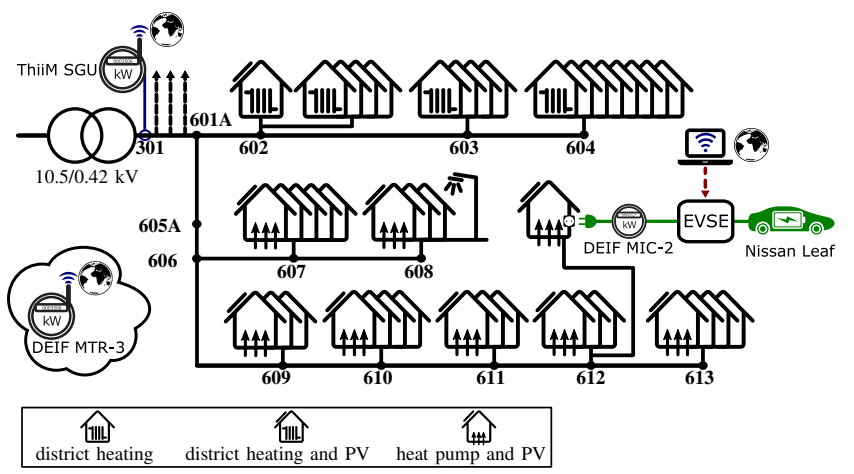

Fig. 3: Schematic overview of the conducted field test and corresponding grid's topology.

(a)

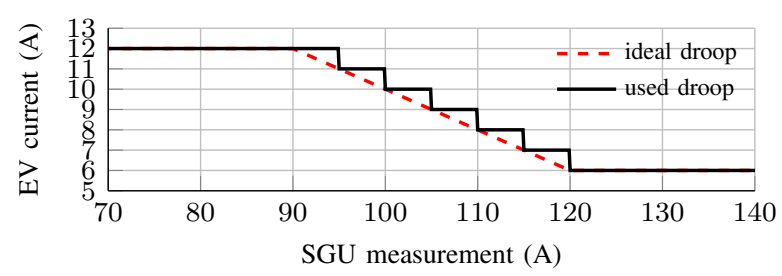

(b)

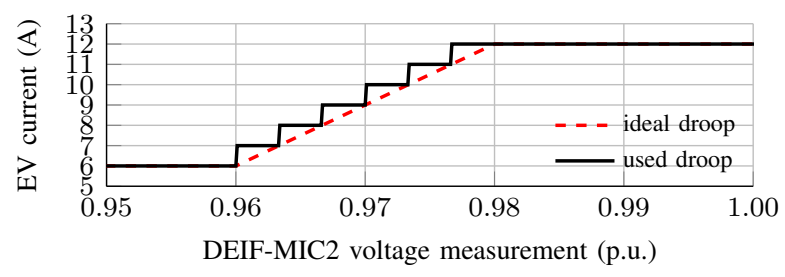

(c)

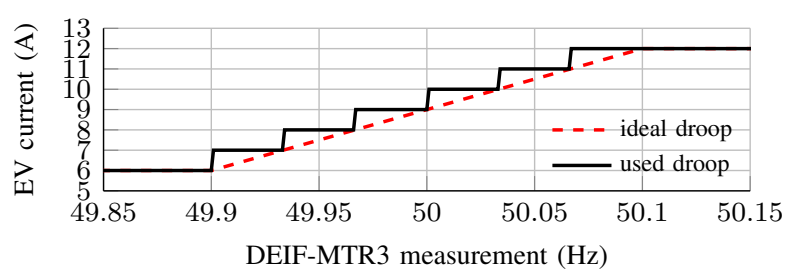

Fig. 4: Implemented droop characteristics for: (a) transformer congestion management, (b) local phase-to-neutral voltage support, and (c) frequency-controlled normal operation reserve.

\section{A. Evaluation criteria}

The conducted test scenarios, whose results are reported in the following section, are respectively: (1) congestion management, (2) local phase-to-neutral voltage support, and (3) frequency-controlled normal reserve in the Nordic synchronous area. Several trials have been conducted for each test scenario, but only selected ones will be reported in detail in Section IV. Regarding the result evaluation for congestion management and voltage support, there are no defined requirements for measurement equipment or response times as such services still do not exist in practise. However, one can assume that if the EV satisfies frequency control requirements, it would also satisfy the future ones for DSO 
services, as the overloading and voltage issues are of much slower nature. FCN requirements only define that all reserve must be supplied within 150 seconds, so for what concerns this manuscript, the EV response time is benchmarked to frequency-controlled disturbance reserve where $50 \%$ of the response must be provided within 5 seconds and the remaining $50 \%$ within additional 25 seconds [33]. The EV performance for each conducted trial is evaluated by assessing several distinctive parameters:

- time difference between the input measurement signal and the set EV current charging limit which will be referred to as control delay,

- time difference between the set EV charging limit and the measured $\mathrm{EV}$ current which will be referred to as $E V$ response time,

- time difference between the input measurement signal and the measured EV current which will be referred to as overall delay, and

- magnitude difference between the set EV charging limit and the measured EV current which will be referred to as EV accuracy.

The evaluated control delay includes the EV charging limit computation time, the communication delay between the control logic and the EVSE controller as well as the time needed for the EVSE controller to change the PWM signal, including the respective measurement delays. The aim is to assess the controller's overall responsiveness and accuracy compared to the ideal droop controllers commonly used in the simulation studies, i.e., the one where the EV responds with no accuracy error and with a negligible response time.

\section{RESULTS}

\section{A. Congestion management}

The first tested ancillary service is congestion management where the $\mathrm{EV}$ is responding to the total feeder current measurement of its respective phase. Fig. 5 shows the measured input, and outputs for one conducted 30-min trial.

First of all, Fig. 5a depicts the total feeder current measurement where two current dips are obvious. These dips correspond to faulty measurements, or more precisely skipped measurement samples which are not an unusual occurrences for measurement units. Since the used measurement device has a 30 second sampling rate, skipped samples result in zero value for half a minute. Secondly, Fig. 5b shows how the EV has the inverse proportional behaviour from the input measurement signal. More precisely, when the feeder current is close to the upper threshold, the EV charges at lower rates, and vice versa. For the skipped measurements, the controller will assume the EV can be charged at maximum rate which may not correspond to reality. However, for validating purposes of this manuscript, the faulty measurements were not seen as an issue, so the resilience to them has not been investigated in detail. A reasonable solution for overcoming these issues could be remaining the previous EV charging limit, which has been left for future work. Finally, it is clear how the measured EV current is not identical to the set charging limit. The shapes of the two curves coincide almost completely, but (a)

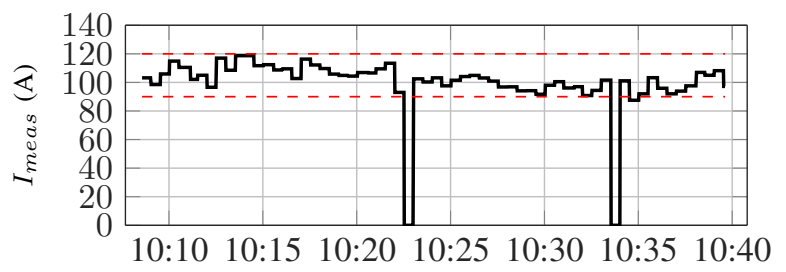

(b)

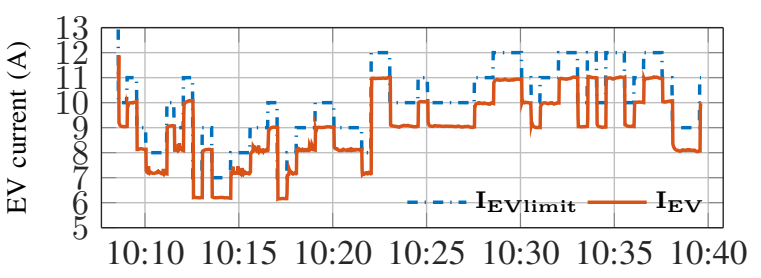

Fig. 5: (a) Measured total feeder current at the transformer station, and (b) set EV charging limit and measured EV response current for the congestion management trial.

there is a consistent offset in their magnitudes. Hence, one can expect that control delay and EV response time are within few seconds, whereas the EV accuracy is not close to the ideal one. The specific values for these parameters are reported in IV-D.

Furthermore, in case of a large EV number, the validated controller can be scaled up and utilised by an aggregator for centralised control. In that case, the whole control logic would be implemented on the aggregator's side, whereas each EVSE would just receive the charging limit as a reference.

\section{B. Local voltage support}

The second tested ancillary service is providing local voltage support, and partially mitigating the EV self-induced low voltages. Contrary to the congestion management trial, the $\mathrm{EV}$ here responds proportionally to the voltage of the phase where it is connected to. In fact, as shown in Fig. 6, the EV charging rate is lower if the measured voltage is low in order not to additionally burden the grid.

(a)

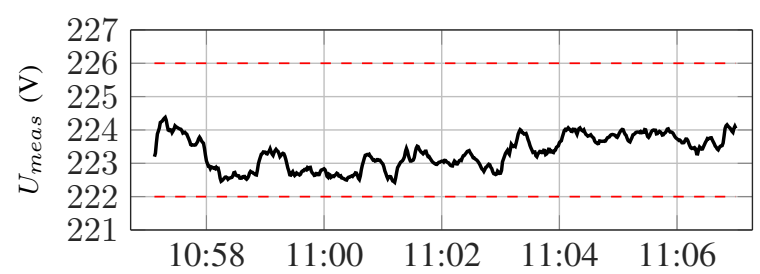

(b)

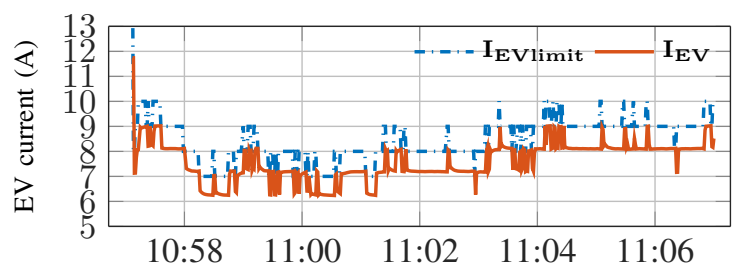

Fig. 6: (a) Measured local phase-to-neutral voltage $U_{c n}$, and (b) set EV charging limit and measured EV response current for voltage support trial. 
Since the validation was conducted on a cold winter day, the residential consumption was relatively high due to heating purposes, resulting in overall low voltages. The measured voltage does not cross the set thresholds, so the EV does not charge at its maximum or minimum rate. Still, since the voltage is not constant, the EV charge is modulated according to the specified droop characteristic. One should note how the chosen droop characteristic is quite steep, and the whole EV flexibility range is utilised within $0.02 U_{n}$ in order to stress the EV by changing the charging limit more often. As the voltage measurements are sampled every second, small voltage deviations result in fast set point changes which can be observed as spikes in Fig. 6, both in the set charging limit, as well as in the measured EV current. Nevertheless, the two curves almost perfectly coincide, and the EV response is not jeopardized by fast changes in the charging limit signal. The accuracy remains similar as in the congestion management trial, which will be discussed later on.

In case of large local EV penetrations, this controller can be used for autonomous voltage support by implementing it within the EVSE, which is already equipped with voltage measurements, and thus reducing ICT costs needed for centralised strategies. For such autonomous control, the service provision can easily be scaled up to larger EV numbers, but once chosen voltage limits could not be remotely changed unless additional communication is implemented. However, specific EVSEs could be given different voltage thresholds by the DSO depending on their connection points, or otherwise, the voltage thresholds could be set to $\pm 10 \% U_{n}$ according to EN50160 requirements [34].

\section{Frequency-controlled normal operation reserve}

The third and final tested ancillary service is providing FCN reserve, whose results are given in Fig. 7. Since the frequency is constantly below $50 \mathrm{~Hz}$ in the observed period, the EV is not modulating the charging rate very often. However, the EV behaviour, both in terms of response time and accuracy, is similar to the one observed in the previous trials.

Utilising decentralised droop controls for providing FCN reserve is a common practice today for large power plants which are equipped with fine frequency meters. Yet, it is highly unlikely to expect the same strategy to be used for EVs since it would imply that each EVSE is equipped with costly high precision frequency measurement device approved by the (a)

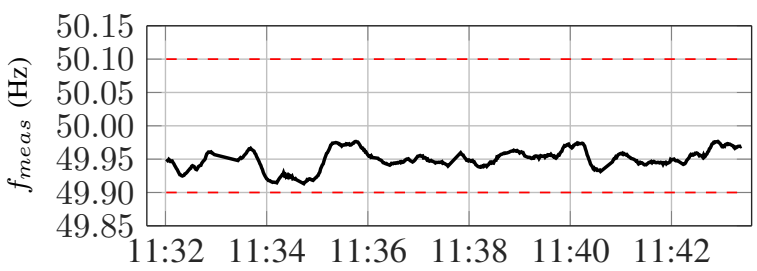

(b)

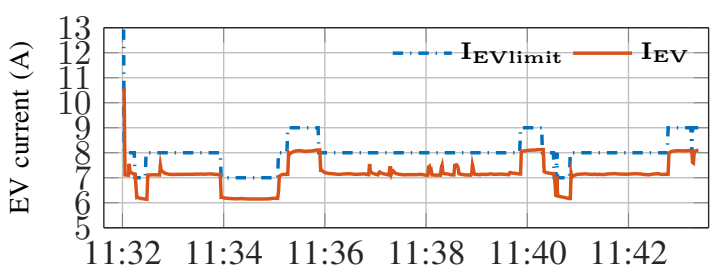

Fig. 7: (a) Measured frequency, and (b) set EV charging limit and measured EV response current for frequency-controlled normal reserve trial.

TSO. Therefore, centralised control concept has been tested in this work, where the frequency measurement is routed via the Internet from a device located $40 \mathrm{~km}$ away at the Technical University of Denmark. Then, only the calculated EV charging limit is sent to the EVSE. However, the tested controller can be utilised for both centralised and decentralised frequency control strategy, since it can be modified to receive local measurements if available, similarly to the voltage support trial.

\section{Result overview and further discussion}

Fig. 8 depicts the relationships between the measured feeder and the measured EV current for one congestion management trial and several overall delays, with the applied droop characteristic highlighted in red. It is clear that the points are more scattered in case of one and three seconds delay, while they are closer to the applied characteristic in case of two seconds delay. Additionally, there is a clear "undershoot" phenomenon in the EV response, as already mentioned in Section IV.

As a measure of the linear dependence degree between two variables, Pearson product-moment (PPM) correlation coefficient is used. This factor ranges between -1 and +1 (a)

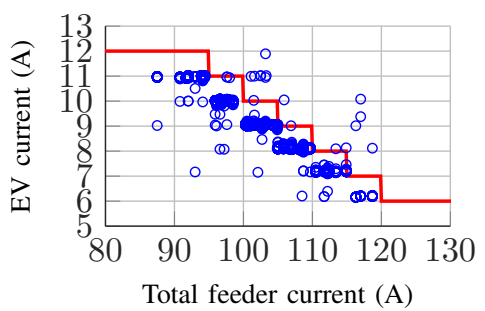

(b)

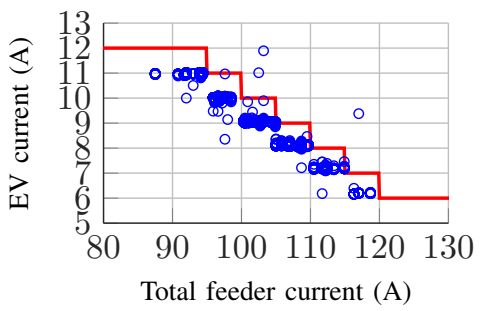

(c)

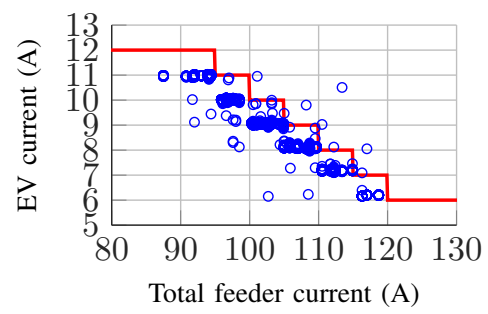

Fig. 8: Relationship between the measured feeder current and the measured EV response current for congestion management field test in case of (a) one second overall delay, (b) two second overall delay, and (c) three second overall delay. 
inclusive, where +1 is the perfect positive correlation (increase in the first variable means increase in the second, and vice versa), and -1 is the perfect negative correlation (increase in the first variable means decrease in the second, and vice versa). Detailed PPM correlation coefficients for all tested ancillary services are reported in Table I as follows:

1) Correlation between the respective input measurement signal, and the EV charging limit set by the controller, used for evaluating the average control delay.

2) Correlation between the EV charging limit set by the controller, and the measured EV current, used for evaluating the average $E V$ response time.

3) Correlation between the respective input measurement signal, and the measured EV current, used for evaluating the average overall delay.

All correlations coefficients are obtained for the data sets excluding the skipped measurement samples.

Several conclusions can be derived from Table I. First of all, the correlation between the input measurement signal $\{I, U, f\}_{\text {meas }}$ and the set $\mathrm{EV}$ charging limit $I_{\text {EVlimit }}$ is the highest for one second delay in all conducted trials, leading to the conclusion that the average control delay is one second. Secondly, even though the highest correlation between the set EV charging limit $I_{E V \text { limit }}$ and the measured EV current $I_{E V}$ is for one second delay in almost all of the trials, it is also comparable for two second delay in the congestion management trials which could be due to the input measurement sampling rate of 30 seconds. In the voltage and frequency trials, where the input signal is sampled every second, the correlation is clearly the highest for one second delay. Therefore, it can be deducted that the average EV response time is one second. Finally, the correlation between the input measurement signal $\{I, U, f\}_{\text {meas }}$ and the measured EV response current $I_{E V}$, which includes all communication and measurement delays, is the highest for two seconds delay, but also comparable for three seconds delay.

Since time is the most critical aspect when providing frequency control, the correlation for different overall delays is shown in Fig. 9 for the FCN reserve trial. It is clear there is no correlation for long time delays, and that the EV response is much faster than the requested 25 seconds. Moreover,

TABLE I: PPM correlation coefficients between input measurements, set EV charging limit and measured EV current for all the tested ancillary services and different $\Delta t$ delays.

\begin{tabular}{cccccc}
\hline \hline & Signals & $\mathbf{\Delta t}=\mathbf{0 s}$ & $\mathbf{\Delta t = 1 s}$ & $\mathbf{\Delta t = 2 s}$ & $\mathbf{\Delta t}=\mathbf{3 s}$ \\
\hline congestion & $I_{\text {meas }}-I_{E V l i m i t}$ & -0.9630 & $\mathbf{- 0 . 9 7 6 8}$ & -0.9635 & -0.9497 \\
management & $I_{E V l i m i t}-I_{E V}$ & 0.9758 & $\mathbf{0 . 9 9 1 3}$ & 0.9904 & 0.9728 \\
trial 01 & $I_{\text {meas }}-I_{E V}$ & -0.9463 & -0.9616 & $\mathbf{- 0 . 9 7 5 4}$ & -0.9713 \\
\hline congestion & $I_{\text {meas }}-I_{E V l i m i t}$ & -0.8758 & $\mathbf{- 0 . 8 7 8 2}$ & -0.8747 & -0.8711 \\
management & $I_{E V l i m i t}-I_{E V}$ & 0.9873 & 0.9935 & $\mathbf{0 . 9 9 3 8}$ & 0.9875 \\
trial 02 & $I_{\text {meas }}-I_{E V}$ & -0.8688 & -0.8728 & $\mathbf{- 0 . 8 7 5 8}$ & -0.8741 \\
\hline voltage & $U_{\text {meas }}-I_{E V l i m i t}$ & 0.8412 & $\mathbf{0 . 9 1 1 9}$ & 0.8950 & 0.8737 \\
support & $I_{E V l i m i t}-I_{E V}$ & 0.8374 & $\mathbf{0 . 9 5 5 7}$ & 0.8605 & 0.7806 \\
trial 01 & $U_{\text {meas }}-I_{E V}$ & 0.8315 & 0.8820 & $\mathbf{0 . 9 2 6 1}$ & 0.9185 \\
\hline voltage & $U_{\text {meas }}-I_{E V l i m i t}$ & 0.7546 & $\mathbf{0 . 8 8 4 3}$ & 0.8805 & 0.8760 \\
suport & $I_{E V l i m i t}-I_{E V}$ & 0.9023 & $\mathbf{0 . 9 2 6 0}$ & 0.8076 & 0.7779 \\
trial 02 & $U_{\text {meas }}-I_{E V}$ & 0.8356 & 0.8575 & $\mathbf{0 . 8 8 3 0}$ & 0.8788 \\
\hline FCN & $f_{\text {meas }}-I_{E V l i m i t}$ & 0.7514 & $\mathbf{0 . 8 8 7 9}$ & 0.8823 & 0.8730 \\
reserve & $I_{E V l i m i t}-I_{E V}$ & 0.8893 & $\mathbf{0 . 9 3 7 7}$ & 0.8191 & 0.7975 \\
trial 01 & $f_{\text {meas }}-I_{E V}$ & 0.8189 & 0.8574 & $\mathbf{0 . 8 9 4 4}$ & 0.8909 \\
\hline \hline
\end{tabular}

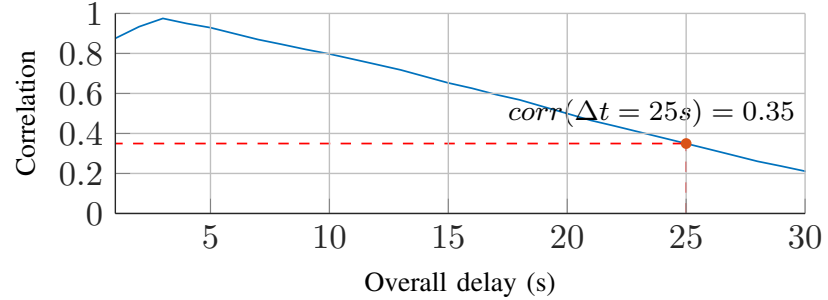

Fig. 9: PPM correlation between the input frequency measurement, and the measured EV current for different overall delays.

TABLE II: Average difference between the set charging limit and the measured EV current - "undershooting" phenomenon.

\begin{tabular}{ccccccc}
\hline \hline $\begin{array}{c}\mathbf{E V} \\
\text { limit }\end{array}$ & $\begin{array}{c}\text { congestion } \\
\text { management } \\
\text { trial 01 }\end{array}$ & $\begin{array}{c}\text { congestion } \\
\text { management } \\
\text { trial 02 }\end{array}$ & $\begin{array}{c}\text { voltage } \\
\text { support } \\
\text { trial 01 }\end{array}$ & $\begin{array}{c}\text { voltage } \\
\text { support } \\
\text { trial 02 }\end{array}$ & $\begin{array}{c}\text { FCN } \\
\text { reserve } \\
\text { trial 01 }\end{array}$ & $\begin{array}{c}\text { all } \\
\text { combined }\end{array}$ \\
\hline $\mathbf{6 ~ A}$ & - & $-0.21 \mathrm{~A}$ & - & - & - & $-0.21 \mathrm{~A}$ \\
$\mathbf{7 ~ A ~}$ & $-0.75 \mathrm{~A}$ & $-0.78 \mathrm{~A}$ & $-0.68 \mathrm{~A}$ & - & $-0.82 \mathrm{~A}$ & $-0.76 \mathrm{~A}$ \\
$\mathbf{8} \mathbf{A}$ & $-0.81 \mathrm{~A}$ & $-0.69 \mathrm{~A}$ & $-0.79 \mathrm{~A}$ & - & $-0.84 \mathrm{~A}$ & $-0.81 \mathrm{~A}$ \\
$\mathbf{9} \mathbf{A}$ & $-0.89 \mathrm{~A}$ & $-0.86 \mathrm{~A}$ & $-0.89 \mathrm{~A}$ & $-0.89 \mathrm{~A}$ & $-0.93 \mathrm{~A}$ & $-0.89 \mathrm{~A}$ \\
$\mathbf{1 0} \mathbf{A}$ & $-0.95 \mathrm{~A}$ & $-0.89 \mathrm{~A}$ & $-1.14 \mathrm{~A}$ & $-1.01 \mathrm{~A}$ & - & $-0.95 \mathrm{~A}$ \\
$\mathbf{1 1} \mathbf{A}$ & $-1.01 \mathrm{~A}$ & $-0.94 \mathrm{~A}$ & - & $-1.25 \mathrm{~A}$ & - & $-0.99 \mathrm{~A}$ \\
$\mathbf{1 2} \mathbf{A}$ & $-1.04 \mathrm{~A}$ & $-1.04 \mathrm{~A}$ & - & - & - & $-1.04 \mathrm{~A}$ \\
\hline \hline
\end{tabular}

by analysing the obtained data, it has been observed that the maximum occurring overall delay equals to four seconds including all Internet communication and measurement delays, which would categorise EVs as a fast reserve. Unless EVs provide a very fast reserve such as the inertial response, there is currently no need for additional requirements to improve the EV response time.

On the contrary, the issue which may occur is not the EV response time, but its accuracy. As aforementioned, the tested EV has far beyond an ideal response, since an "undershooting" phenomenon occurs. The average difference between the set charging limit $I_{E \text { Vlimit }}$ and the measured current $I_{E V}$ is given in Table II. Interestingly, the higher the set charging limit is, the more does the EV "undershoot", leading up to over $1 \mathrm{~A}$ difference for the $12 \mathrm{~A}$ charging limit. There could be several reasons to explain this phenomenon. First of all, contemporary standards define that EVs must be able to respond to the charging limit, and guarantee that the EV is charging below it. However, one must emphasize that they do not define what is the acceptable deviation from the set limit, so EVs are not manufactured to respond as close as possible to it. Secondly, the EV battery management system is highly dependent on many factors, and the authors believe one of the factors is the outside temperature. In fact, in occasion of previous experiments in laboratory environment [22], the "undershooting" phenomenon was lower than for the conducted field test performed on a winter day with temperatures below $0^{\circ} \mathrm{C}$. Thirdly, the battery management system may also be influenced by the battery state of charge (SOC) and previous driving behaviour. Unfortunately, these hypotheses cannot be thoroughly investigated as the information from the battery management system itself is not broadly available.

As a final remark, modulating the EV active power 
influences the SOC and consequently user comfort and, in the worst case, the EV would constantly charge at the minimum $6 \mathrm{~A}$. According to [35], the average EV plug-in time is $13 \mathrm{~h}$, whereas the initial SOC equals to around 50\%. Assuming the battery size of $24 \mathrm{kWh}$, the EV charging time would increase from around 3.5 hours at a $16 \mathrm{~A}$ charging rate to around 8.5 hours at a $6 \mathrm{~A}$ rate, which is still well below the average plug-in time of 13 hours. However, due to many uncertainties, the authors are aware that EV owners may not allow active power modulation due to fear of not having the EV available for transportation purposes. In that case, the same principle could be used for modulating the EV reactive power which does not influence the SOC. However, current EVs are not equipped with reactive power control, so future work includes applying this controller for modulating the power factor when EVs will be capable of it

\section{CONCLUSION}

This paper focused on validating the technical feasibility of a series-produced EV to provide flexibility in real distribution grids. It presents a droop controller which uses contemporary standards and can be used with all series-produced EVs complying with international standards IEC 61851 and SAE J1772. The conducted field validation tested three ancillary services: congestion management, local voltage support and frequency-controlled normal operating reserve. Overall, the field validation proved that providing ancillary services by EVs is technically feasible already today with existing commercial EVs without any Vehicle-to-Grid capability, and with a very fast response time. The overall delay, including all communication and measurement delays, was 2-3 seconds in average, and never exceeded 4 seconds. However, an "undershooting" phenomenon in current magnitude was noticed when limiting the EV charging rate which may arise as a greater problem than the response time. This difference varied depending on the set charging limit, but can amount to more than $1 \mathrm{~A}$.

There is much room for improvement in EV integration, and the authors have identified several points. First of all, the EV charging systems should not be designed only to guarantee the charging current below a certain limit, but also to be as close as possible to the preferred limit. Considering the available 1 A granularity, an "undershooting" of 1 A can be considered unacceptable as it corresponds to a lower charging set point. Secondly, the overall delay is currently more than enough for the distribution grid services, but it could be additionally shortened by optimising control, communication and EV charging system. This could be of particular value for several services, such as frequency control or provision of virtual inertia. Finally, the granularity of $1 \mathrm{~A}$ may not be good enough for using EVs for smart grid purposes, since $1 \mathrm{~A}$ amounts to $10 \%$ of EV's available flexibility. Considering that EVs are high loads and have a significant grid impact, lower granularity would provide a higher flexibility degree with potentially less influence on the EV owners. More precisely, it would allow EVs to charge at an intermediate rate low enough to mitigate the grid adverse effects, but as high as possible to charge the vehicle faster.
Since EVs could be a valuable asset for all power system entities, the authors' believe that additional standards are needed to address the identified issues, and oblige the EV manufactures to optimise their systems. Future work includes the extension of the field testing to several vehicles to assess the coordination issues, as well as extending the controller logic to more advanced distributed approaches.

\section{ACKNOWLEDGMENT}

This work is supported by the Danish Research Project "NIKOLA - Intelligent Electric Vehicle Integration" under ForskEL kontrakt nr. 2013-1-12088. More information at www.nikolaproject.info.

\section{REFERENCES}

[1] J. Y. Yong, V. K. Ramachandaramurthy, K. M.Tan and N. Mithulananthan, "A review on the state-of-the-art technologies of electric vehicle, its impacts and prospects," Renewable and Sustainable Energy Reviews, vol. 49, pp. 365 - 385, 2015.

[2] E. Grunditz and T. Thiringer, "Performance Analysis of Current BEVs - Based on a Comprehensive Review of Specifications," IEEE Transactions on Transportation Electrification, vol. PP, no. 99, pp. 1-1, 2016.

[3] N. S. Pearre, W. Kempton, R. L. Guensler, and V. V. Elango, "Electric vehicles: How much range is required for a day's driving?" Transportation Research Part C: Emerging Technologies, vol. 19, no. 6, pp. 1171-1184, 2011.

[4] P. Andersen, M. Marinelli, O. Olesen, C. Andersen, G. Poilasne, B. Christensen, and O. Alm, "The Nikola project Intelligent electric vehicle integration," in Innovative Smart Grid Technologies Conference Europe (ISGT-Europe), 2014 IEEE PES, Oct 2014.

[5] J. García-Villalobos, I. Zamora, J. I. San Martín, F. J. Asensio, and V. Aperribay, "Plug-in electric vehicles in electric distribution networks: A review of smart charging approaches," Renewable and Sustainable Energy Reviews, vol. 38, pp. 717-731, 2014.

[6] J. Xiong, K. Zhang, Y. Guo, and W. Su, "Investigate the impacts of PEV charging facilities on integrated electric distribution system and electrified transportation system," IEEE Transactions on Transportation Electrification, vol. 1, no. 2, pp. 178-187, Aug 2015.

[7] W. Kempton and J. Tomić, "Vehicle-to-grid power implementation: From stabilizing the grid to supporting large-scale renewable energy," Journal of Power Sources, vol. 144, pp. 280-294, 2005.

[8] J. Meng, Y. Mu, H. Jia, J. Wu, X. Yu, and B. Qu, "Dynamic frequency response from electric vehicles considering travelling behavior in the Great Britain power system," Applied Energy, vol. 162, pp. 966 - 979, 2016.

[9] P. R. Almeida, F. Soares, and J. P. Lopes, "Electric vehicles contribution for frequency control with inertial emulation," Electric Power Systems Research, vol. 127, pp. 141 - 150, 2015.

[10] H. Liu, Z. Hu, Y. Song, and J. Lin, "Decentralized vehicle-to-grid control for primary frequency regulation considering charging demands," IEEE Transactions on Power Systems, vol. 28, no. 3, pp. 3480-3489, Aug 2013.

[11] K. Knezović, M. Marinelli, P. Codani, and Y. Perez, "Distribution grid services and flexibility provision by electric vehicles: A review of options," in Power Engineering Conference (UPEC), 2015 50th International Universities, Sept 2015, pp. 1-6.

[12] E. Niesten and F. Alkemade, "How is value created and captured in smart grids? A review of the literature and an analysis of pilot projects," Renewable and Sustainable Energy Reviews, vol. 53, pp. 629 - 638 , 2016.

[13] J. Hu, C. Si, M. Lind, and R. Yu, "Preventing distribution grid congestion by integrating indirect control in a hierarchical electric vehicles management system," IEEE Transactions on Transportation Electrification, vol. PP, no. 99, 2016.

[14] O. Sundstrom and C. Binding, "Flexible charging optimization for electric vehicles considering distribution grid constraints," IEEE Transactions on Smart Grid, vol. 3, no. 1, pp. 26-37, March 2012.

[15] P. Richardson, D. Flynn, and A. Keane, "Local versus centralized charging strategies for electric vehicles in low voltage distribution systems," IEEE Transactions on Smart Grid, vol. 3, no. 2, pp. 1020-1028, June 2012. 
[16] N. Leemput, F. Geth, J. Van Roy, A. Delnooz, J. Buscher, and J. Driesen, "Impact of electric vehicle on-board single-phase charging strategies on a Flemish residential grid," IEEE Transactions on Smart Grid, vol. 5, no. 4, pp. 1815-1822, July 2014.

[17] K. Knezović and M. Marinelli, "Phase-wise enhanced voltage support from electric vehicles in a danish low-voltage distribution grid," Electric Power Systems Research, vol. 140, pp. 274 - 283, 2016.

[18] N. Leemput, F. Geth, J. V. Roy, J. Büscher, and J. Driesen, "Reactive power support in residential LV distribution grids through electric vehicle charging," Sustainable Energy, Grids and Networks, vol. 3, pp. $24-35,2015$.

[19] R. Abousleiman and R. Scholer, "Smart charging: System design and implementation for interaction between plug-in electric vehicles and the power grid," IEEE Transactions on Transportation Electrification, vol. 1, no. 1, pp. 18-25, June 2015.

[20] P. C. Kotsampopoulos, F. Lehfuss, G. F. Lauss, B. Bletterie, and N. D. Hatziargyriou, "The limitations of digital simulation and the advantages of PHIL testing in studying distributed generation provision of ancillary services," IEEE Transactions on Industrial Electronics, vol. 62, no. 9, pp. 5502-5515, Sept 2015.

[21] C. Gouveia, C. L. Moreira, J. A. P. Lopes, D. Varajao, and R. E. Araujo, "Microgrid service restoration: The role of plugged-in electric vehicles,' IEEE Industrial Electronics Magazine, vol. 7, no. 4, pp. 26-41, Dec 2013.

[22] M. Marinelli, S. Martinenas, K. Knezović, and P. B. Andersen, "Validating a centralized approach to primary frequency control with series-produced electric vehicles," Journal of Energy Storage, vol. 7, pp. $63-73,2016$

[23] IEC 61851-1:2010, "Electric vehicle conductive charging system - Part 1: General requirements," 2010.

[24] SAE J1772:2010, "Electric vehicle and plug in hybrid electric vehicle conductive charge coupler," 2010.

[25] Danish Electric Vehicle Association, "Stock information for electric vehicles in Denmark," 2016. [Online]. Available: http://www.danskelbilalliance.dk/Statistik

[26] S. Izadkhast, P. Garcia-Gonzalez and P. Frías, "An Aggregate Model of Plug-In Electric Vehicles for Primary Frequency Control," IEEE Transactions on Power Systems, vol. 30, no. 3, pp. 1475-1482, May 2015.

[27] J. R. Pillai and B. Bak-Jensen, "Vehicle-to-Grid for islanded power system operation in Bornholm," in IEEE PES General Meeting, July 2010, pp. $1-8$

[28] J. A. Peças Lopes, S. A. Polenz, C. L. Moreira and R. Cherkaoui, "Identification of control and management strategies for LV unbalanced microgrids with plugged-in electric vehicles," Electric Power Systems Research, vol. 80, no. 8, pp. 898 - 906, 2010.

[29] S. Martinenas, K. Knezović, and M. Marinelli, "Management of power quality issues in low voltage networks using electric vehicles: Experimental validation," IEEE Transactions on Power Delivery, vol. PP, no. 99, pp. 1-9, 2016.

[30] S. Mocci, N. Natale, F. Pilo and S. Ruggeri, "Demand side integration in LV smart grids with multi-agent control system," Electric Power Systems Research, vol. 125 , pp. 23 - 33, 2015

[31] J. Hu, A. Saleem, S. You, L. Nordström, M. Lind and J. Østergaard, “A multi-agent system for distribution grid congestion management with electric vehicles," Engineering Applications of Artificial Intelligence, vol. 38 , pp. $45-58,2015$.

[32] H. N. T. Nguyen, C. Zhang, and J. Zhang, "Dynamic demand control of electric vehicles to support power grid with high penetration level of renewable energy," IEEE Transactions on Transportation Electrification, vol. 2, no. 1, pp. 66-75, March 2016.

[33] Energinet.dk, "Ancillary services to be delivered in Denmark - Tender conditions," 2012

[34] EN 50160:2010, "Voltage characteristics of electricity supplied by public electricity networks," 2010.

[35] P. Andersen, "Intelligent electric vehicle integration - domain interfaces and supporting informatics," Ph.D. dissertation, Technical University of Denmark, 2013.

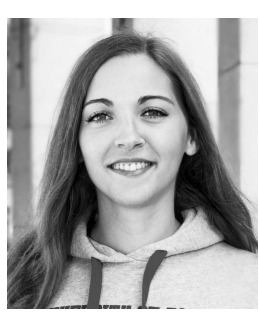

Katarina Knezović (S'13) was born in Zagreb, Croatia, in 1989. She has received her B.Sc. and M.Sc. degrees in electrical engineering from the University of Zagreb, Croatia, in 2011 and 2013 respectively. She is currently pursuing a $\mathrm{Ph} . \mathrm{D}$. degree in electrical engineering at the Technical University of Denmark.

Her research interests include power-system modeling, distribution networks, and grid-coupling of electric vehicles.

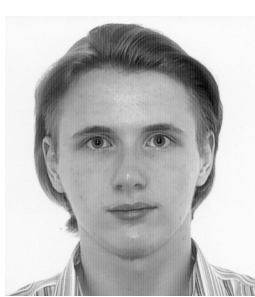

Sergejus Martinenas (S'14) was born in Elektrenai, Lithuania, in 1989. He received a B.Sc. degree in mechatronics engineering from the University of Southern Denmark in 2011, and an M.Sc. degree in electrical engineering from the Technical University of Denmark in 2014. He is currently pursuing the Ph.D. degree in electrical engineering at DTU.

His research focuses on enabling technologies for electric vehicle integration into smart grids.

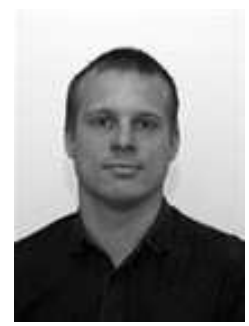

Peter Bach Andersen (S'13) was born in Copenhagen in 1982. He holds a Ph.D. from the Technical University of Denmark. His main focus is the application of informatics for electric vehicle integration. This includes software, communication technology, data analysis, and optimization.

Peter is currently managing a number of research projects and initiatives, including running the EV Lab dedicated to this line of research.

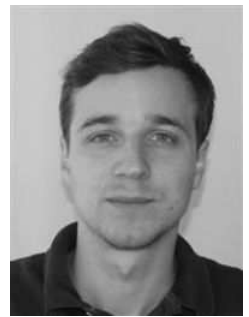

Antonio Zecchino was born in Padova, Italy, on July the 4th, 1990. He received the B.Sc. degree in energy engineering and the M.Sc. degree in electrical engineering from the University of Padova in 2012 and 2015, respectively. He is currently Ph.D. student at the Center for Electric Powe and Energy at the Technical University of Denmark (DTU)

His research interests include electric vehicles' integration in the power system, investigation of grid services provision from electric vehicles to support the power grid, and distribution grid modeling, simulation and testing

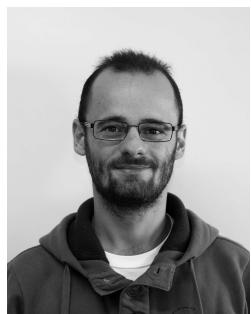

Mattia Marinelli (S'10-M'12) was born in Genova, Italy, in 1983. He received B.Sc. and M.Sc. degrees in electrical engineering from the University of Genova in 2005 and 2007. In March 2011 he achieved the European Ph.D. degree in power systems. Since September 2012 he has been with the Technical University of Denmark.

His research regards power system integration studies, wind and solar data analysis, electric vehicles and distributed energy resources modeling. 